\title{
Khintchine's Theorem and Approximation of Zero by the Values of Integer Polynomials in Different Metrics
}

\author{
N. V. Budarina ${ }^{a}$, D. Dickinson ${ }^{b}$, and V. I. Bernik ${ }^{c}$ \\ Presented by Academician V.V. Kozlov June 19, 2006
}

Received November 23, 2006

\section{DOI: $10.1134 / \mathrm{S} 106456240702007 \mathrm{X}$}

The rate of approximation of real numbers by rationals allows one to draw many conclusions on the arithmetic nature of numbers. In this way, the existence of transcendental numbers was first proved, the theory of Thue equations was constructed, the irrationality of $\zeta(3)$ was shown, etc. However, some problems remain unsolved. At the same time, real numbers are amazingly similar from the point of view of measure theory. It was noted by Borel, and Khintchine [1] proved his famous theorem. Let $\Psi(x)$ be a monotonically decreasing function defined on $\mathbf{R}_{+}, \mu A$ be the Lebesgue measure of a measurable set $A \subset \mathbf{R}$, and $I=[a, b]$ be an interval.

Khintchine's theorem. Let $L_{1}(\Psi)$ be the set of real numbers $x \in$ I for which the inequality

$$
\left|x-\frac{p}{q}\right|<\frac{\Psi(q)}{q}
$$

has infinitely many solutions in integers $p$ and positive integers $q$. Then

$$
\mu L_{1}(\Psi)=\left\{\begin{array}{llc}
0, & \text { if } & \sum_{q=1}^{\infty} \Psi(q)<\infty, \\
\mu I, & \text { if } & \sum_{q=1}^{\infty} \Psi(q)=\infty .
\end{array}\right.
$$

\footnotetext{
${ }^{a}$ Vladimir State Pedagogical University, pr. Stroitelei 11, Vladimir, 600024 Russia

e-mail: budarina@vgpu.vladimir.ru

${ }^{b}$ NUI Maynooth, Maynooth, Co. Kildare, Ireland e-mail:ddickinson@maths.nuim.ie

${ }^{c}$ Institute of Mathematics, Belarussian Academy of Sciences, ul. Surganova 11, Minsk, 220072 Belarus

e-mail:bernik@im.bas-net.by
}

If the series $\sum_{q=1}^{\infty} \Psi(q)$ converges, the monotonicity assumption for $\Psi(q)$ can be dropped; otherwise, the theorem does not hold without this assumption. Inequality (1) can be rewritten as

$$
|q x-p|<\Psi(q) .
$$

Then we deal with the solvability of inequality (2) in first-degree polynomials with integer coefficients. Let

$$
\begin{gathered}
P_{n}(x)=a_{n} x^{n}+a_{n-1} x^{n-1}+\ldots+a_{1} x+a_{0}, \\
a_{j} \in Z, 0 \leq j \leq n, H=H(P)=\max _{1 \leq i \leq n}\left|a_{i}\right| .
\end{gathered}
$$

Denote by $L_{n}(\Psi)$ the set of $x \in \mathbf{R}$ for which the inequality

$$
\left|P_{n}(x)\right|<H^{-n+1} \Psi(H)
$$

has infinitely many solutions in integer polynomials $P(x)$. The problem of the measure of $L_{n}(\Psi)$ has a long history. Specifically, in 1932 Mahler [2] conjectured that $L_{n}(\Psi)$ has measure zero for $\Psi(H)=H^{-\lambda}$, where $\lambda>1$. His conjecture was proved by Sprindzhuk [3]. Baker [4] somewhat improved Sprindzhuk's result and conjectured that Khintchine's theorem holds for $L_{n}(\Psi)$ in the case of convergence. This conjecture was proved in [5], and, in the case of divergence, in [6]. Soon afterwards, these results were extended to the fields of complex and $p$-adic numbers $[7,8]$.

This work deals with simultaneous approximation of zero in all three metrics. Hereafter, $\mu_{1}$ is the Lebesgue measure in $\mathbf{R}, \mu_{2}$ is the measure in the field of complex numbers, $\mu_{3}$ is the measure in $Q_{p}$, and $\mu=\mu_{1} \times$ $\mu_{2} \times \mu_{3}$. Let $\overline{\boldsymbol{v}}=\left(v_{1}, v_{2}, v_{3}\right)$ and $\bar{\lambda}=\left(\lambda_{1}, \lambda_{2}, \lambda_{3}\right)$ be vectors with positive real coordinates, and let $T=I \times K \times D$ be a parallelepiped in $\mathbf{R} \times C \times Q$, where $I \subset \mathbf{R}$ is an interval, $K \subset C$ is a disk in $C$, and $D$ is a cylinder in $Q_{p}$. 
Denote by $\operatorname{Ln}(\overline{\mathbf{v}}, \bar{\lambda}, \Psi)$ the set of $\overline{\mathbf{u}}=(x, z, w) \in T$ for which the system of inequalities

$$
\begin{gathered}
|P(x)|<H^{-v_{1}} \Psi^{\lambda_{1}}(H), \quad|P(z)|<H^{-v_{2}} \Psi^{\lambda_{2}}(H), \\
|P(w)|_{p}<H^{-v_{3}} \Psi^{\lambda_{3}}(H), \\
v_{1}+2 v_{2}+v_{3}=n-3, \quad \lambda_{1}+2 \lambda_{2}+\lambda_{3}=1,
\end{gathered}
$$

has infinitely many solutions in polynomials (3).

Theorem 1. Under conditions (5) and $n \geq 3$,

$$
\mu L_{n}(\overline{\mathbf{v}}, \bar{\lambda}, \Psi)=0
$$

if $\sum_{H=1}^{\infty} \Psi(H)<\infty$.

Theorem 2. Under conditions (5), $n \geq 3, v_{1}+1=$ $v_{2}+1=v_{3}$, and $\lambda_{1}=\lambda_{2}=\lambda_{3}$,

$$
\mu L_{n}(\overline{\mathbf{v}}, \bar{\lambda}, \Psi)=\mu T,
$$

if $\sum_{H=1}^{\infty} \Psi(H)=\infty$

Remark 1. The conditions imposed on $\overline{\mathbf{v}}$ and $\overline{\boldsymbol{\lambda}}$ are not final. They cover the most interesting range of $\overline{\mathbf{v}}$ and $\bar{\lambda}$ and make it possible to explain the idea behind the proof of the theorem.

Remark 2. In the circle $K$ for given $\delta>0$, we do not need to consider the complex numbers $z$ with $|\operatorname{Im} z| \leq \delta$ since the measure of such $z$ is small.

Proof sketch of Theorem 1. The standard technique in the proof is the transition in (5) to irreducible polynomials with the conditions

$$
\left|a_{n}\right|=H, \quad\left|a_{n}\right|_{p}>\frac{1}{p^{n}} .
$$

In what follows, $\varepsilon>0$ is a sufficiently small real number, $T=T(\varepsilon, n)$ is a sufficiently large positive integer, and $\varepsilon_{1}=\frac{\varepsilon}{T}$. Consider a vector $\bar{\xi}=\left(\alpha_{1}, \beta_{1}, \gamma_{1}\right)$, where $P\left(\alpha_{1}\right)=P\left(\beta_{1}\right)=P\left(\gamma_{1}\right)=0, \alpha_{1} \in \mathbf{R}, \beta_{1} \in C$, and $\gamma_{1} \in Q_{p}$. The remaining roots are arranged according to their distances to $\alpha_{1}, \beta_{1}$, and $\gamma_{1}$, respectively.

We solve the equations $\left|\alpha_{1}-\alpha_{j}\right|=H^{-\mu_{1 j}},\left|\beta_{1}-\beta_{j}\right|=$ $H^{-\mu_{2 j}}$, and $\left|\gamma_{1}-\gamma_{j}\right|=H^{-\mu_{3 j}}(2 \leq j \leq n)$ and find integers $k_{j}$, $l_{j}$, and $m_{j}$ such that $\frac{k_{j}-1}{T} \leq \mu_{1 j}<\frac{k_{j}}{T}, \frac{l_{j}-1}{T} \leq \mu_{2 j}<\frac{l_{j}}{T}$, and
$\frac{m_{j}-1}{T} \leq \mu_{3 j}<\frac{m_{j}}{T}$. Define $q_{i}=\frac{1}{T} \sum_{j=i+1}^{n} k_{j}, r_{i}=$ $\frac{1}{T} \sum_{j=i+1}^{n} l_{j}$, and $s_{i}=\frac{1}{T} \sum_{j=i+1}^{n} m_{j}$.

An important point in the proof is the relation between $\overline{\mathbf{d}}=\left(q_{1}+\frac{k_{2}}{T}, r_{1}+\frac{l_{2}}{T}, s_{1}+\frac{m_{1}}{T}\right)$ and $\overline{\mathbf{e}}=\left(v_{1}+\right.$ $\lambda_{1}, v_{2}+\lambda_{2}, v_{3}+\lambda_{3}$ ). If all the coordinates of $\overline{\mathbf{d}}$ are smaller than those of $\overline{\mathbf{e}}$, then the system of inequalities

(5) in the neighborhood of $\bar{\xi}$ is called $(0,0,0)$-linear; otherwise, it is called $(1,1,1)$-linear. Clearly, there are another six types of linearity. How to deal with these two types of linearity can be explained as follows. Denote by $F_{n}$ the class of irreducible polynomials with

condition (6), and let $F_{t}=\bigcup_{2^{t} \leq H<2^{t+1}} P_{n}$. Define $d_{1}=q_{1}+$ $2 r_{1}+s_{1}$ and $d_{2}=\frac{k_{2}+2 l_{2}+m_{2}}{T}$, and let $d_{1}+d_{2}>n+\varepsilon$.

From the inequalities for polynomials in (5), we can proceed to estimates of $\left|x-\alpha_{1}\right|,\left|z-\beta_{1}\right|$, and $\left|w-\gamma_{1}\right|_{p}$ by using well-known inequalities $[3,8]$. On any of the parallelepipeds $M=2^{-t k_{2}} \times 2^{-t l_{2}} \times 2^{-t m_{2}}$, where system (5) is solvable for $P(f)$ with $f=(x, z, w)$, the polynomial $P(f)$ in expanded in a Taylor series and all the expansion terms are estimated from above to obtain

$$
\begin{gathered}
|P(x)|<c(n) \times 2^{-t\left(q_{1}+k_{2} T^{-1}-1-(n-1) \varepsilon_{1}\right)}, \\
|P(z)|<c(n) \times 2^{-t\left(r_{1}+l_{2} T^{-1}-1-(n-1) \varepsilon_{1}\right)}, \\
|P(w)|_{p}<c(n) \times 2^{-t\left(s_{1}+m_{2} T^{-1}-(n-1) \varepsilon_{1}\right)} .
\end{gathered}
$$

According to [9], the existence of more than one polynomial on $M$ with condition (5) leads to a contradiction. If there is no more than one polynomial on $M$, the required estimate is derived by direct calculation.

The case $4-\varepsilon<d_{1}+d_{2} \leq n+\varepsilon$ is the key point in the proof. Once again, $T$ is divided into parallelepipeds $M=$ $2^{-t k_{2} T^{-1}} \times 2^{-t l_{2} T^{-1}} \times 2^{-t m_{2} T^{-1}}$, is expanded in a Taylor series, and all the polynomials with condition (5) are estimated on $M$. First, consider only those $M$ that contain no more than $c(n) \times 2^{t \theta}$ polynomials. Direct calculations show that there exists a bound for $\theta$ below which the theorem holds true. If $\theta$ exceeds this bound, then, using the Dirichlet principle and subtracting the polynomials of degree $n$, we obtain lower degree polynomials. As a result, we derive a system of form (7) with polynomials $R(f)$, where $\operatorname{deg} R \leq n$. For low-degree polynomials, Theorem 1 can be proved by direct calculation. 
For $(1,1,1)$-linearity, the proof is close to that for $d_{1}+d_{2}>n+\varepsilon$, but $T$ is divided into smaller parallelepipeds.

To prove Theorem 2 on divergence, we need the socalled optimal regular system. It is constructed using the Dirichlet principle, applying which we can easily prove that, for any $Q>1$ and any $\overline{\mathbf{u}} \in T$, there exists $c_{1}>$ 0 and a polynomial $P(f)$ that is not identically zero such that

$$
\begin{array}{ll}
|P(x)|<c_{1} Q^{-w_{1}}, & \\
|P(z)|<c_{1} Q^{-w_{2}}, & w_{1}+2 w_{2}+w_{3} \leq n-2, \\
|P(w)|_{p}<c_{1} Q^{-w_{3}}, & H(P) \leq Q, \quad \operatorname{deg} P \leq n .
\end{array}
$$

The basic difficulty is to prove the existence of $c_{2}>$ 0 for which, along with (8), the inequality

$$
\max \left(\left|P^{\prime}(x)\right|,\left|P^{\prime}(z)\right|, Q\left|P^{\prime}(w)\right|_{p}\right)>c_{2} Q
$$

holds for $f \in B \subset T, \mu B>\frac{\mu T}{2}$. We show that, if (9) is violated, $f$ belongs to a set of small measure. If (8) and (9) are satisfied, it can be shown that there exists a vec- tor $\bar{\xi}=(\alpha, \beta, \gamma)$ with $P(\alpha)=P(\beta)=P(\gamma)=0, \alpha \in \mathbf{R}$, $\beta \in C$, and $\gamma \in Q_{p}$ such that ||$x-\alpha\left|=c(n) H^{-w_{1}-1},\right| z-\beta \mid=$ $c(n) H^{-w_{2}-1}$, and $|w-\gamma|_{p}=H^{-w_{3}}$. From the set of all roots $\bar{\xi}$, we choose the maximum system with a given distance between different $\bar{\xi}$. This system is an optimal regular one. The rest of the proof is the same as in [6].

\section{REFERENCES}

1. A. Khintchine, Math. Ann. 92, 115-125 (1924).

2. K. Mahler, Math. Ann. 105, 131-139 (1932).

3. V. G. Sprindzhuk, Izv. Akad. Nauk SSSR, Ser. Mat. 29, 379-436 (1965).

4. A. Baker, Proc. R. Soc. London A 292, 92-104 (1966).

5. V. Bernik, Acta Arithm. 53, 17-28 (1989).

6. V. V. Beresnevich, Acta Arithm. 90, 97-112 (1999).

7. V. I. Bernik and D. V. Vasil'ev, Tr. Inst. Mat. Nats. Akad. Nauk Belarusi 3, 10-20 (1999).

8. E. I. Kovalevskaya, Preprint No. 8 (547) IM NAN Belarusi (Inst. of Mathematics, National Academy of Sciences of Belarus, 1998).

9. V. I. Bernik and N. I. Kalosha, Vestsi Nats. Akad. Nauk Belarusi, Ser. Fiz. Mat., No. 1, 121-123 (2004). 